\title{
THE SPECTRUM OF A PARAMETRIZED PARTIAL DIFFERENTIAL OPERATOR OCCURRING IN HYDRODYNAMICS
}

\author{
R. DENK, M. MÖLLER, AND C. TRETTER
}

\begin{abstract}
A partial differential operator associated with natural oscillations of an incompressible fluid in the neighbourhood of an elliptical flow is considered. The differentiation is only taken with respect to the angular variable, and thus the operator becomes a family of ordinary differential operators parametrized by the radial variable. It is shown that the spectra of these ordinary differential operators completely determine the spectrum of the given operator which turns out to have a kind of skeleton structure.
\end{abstract}

\section{INTRODUCTION}

The analysis of natural oscillations of an incompressible fluid in the neighbourhood of an elliptical flow leads to a spectral problem for an operator $\mathfrak{A}$ of the form (see Lifschitz [3])

$$
\mathfrak{A}=\left(\begin{array}{cc}
\frac{\partial}{\partial \psi} & 0 \\
0 & \frac{\partial}{\partial \psi}
\end{array}\right)+2 N
$$

in $L^{2}((0, \infty) \times(0,2 \pi), \rho)^{2}$ with (see $\left.[3,(20)]\right)$

$$
N(\rho, \psi)=\left(\begin{array}{cc}
0 & -\frac{1-\delta^{2}}{\rho^{2}(1-\delta \cos (2 \psi))+1-\delta^{2}} \\
1 & \frac{\delta \rho^{2} \sin (2 \psi)}{\rho^{2}(1-\delta \cos (2 \psi))+1-\delta^{2}}
\end{array}\right), \quad \rho \in(0, \infty), \psi \in(0,2 \pi),
$$

where $(\rho, \psi)$ are polar coordinates. The domain of $\mathfrak{A}$ is given by

$$
\begin{aligned}
D(\mathfrak{A})=\left\{f \in L^{2}((0, \infty) \times(0,2 \pi), \rho)^{2}:\right. & f(\rho, \cdot) \in H^{1}(0,2 \pi)^{2} \text { for almost all } \rho, \\
& f(\cdot, 0)=f(\cdot, 2 \pi)\} .
\end{aligned}
$$

The ellipticity parameter $\delta \in[0,1)$ characterizes the geometry of the flow; for the special case $\delta=0$ the flow becomes circular.

The operator $\mathfrak{A}$ can also be considered in the space $L^{2}\left((0, \infty), \rho, L^{2}(0,2 \pi)^{2}\right)$ consisting of all $L^{2}$-functions on $(0, \infty)$ with respect to the weight $\rho$ having values in $L^{2}(0,2 \pi)^{2}$. In this space the domain of $\mathfrak{A}$ is given by

$$
D(\mathfrak{A})=\left\{f \in L^{2}\left((0, \infty), \rho, H^{1}(0,2 \pi)^{2}\right): f(\rho)(0)=f(\rho)(2 \pi) \text { for almost all } \rho\right\} .
$$

AMS Subj. Class.: Primary 47F05; secondary 47E05, 76B99 
In this setting, the partial differential operator $\mathfrak{A}$ turns out to be related to the family of ordinary differential operators (compare [3, pp. 1627-1628])

$$
A(\rho)=\left(\begin{array}{cc}
\frac{d}{d \psi} & 0 \\
0 & \frac{d}{d \psi}
\end{array}\right)+2 N(\rho, \cdot), \quad \rho \in(0, \infty),
$$

acting in the space $L^{2}(0,2 \pi)^{2}$ with domain

$$
D:=D(A(\rho))=\left\{f(\rho) \in H^{1}(0,2 \pi)^{2}: f(\rho)(0)=f(\rho)(2 \pi)\right\}
$$

not depending on $\rho$. This relation is given by

$$
(\mathfrak{A} f)(\rho)=A(\rho) f(\rho), \quad \rho \in(0, \infty) .
$$

Hence $\mathfrak{A}$ can be considered as a generalized multiplication operator. However, since $\mathfrak{A}$ is not self-adjoint, the spectrum of $\mathfrak{A}$ cannot be determined using the results of Reed and Simon [5, Section XIII.16].

In the next section we are going to show that the spectrum of $\mathfrak{A}$ is the union of the spectra of $A(\rho)$ taken over $\rho \in[0, \infty]$. In the following Section 3 we calculate the spectrum of $\mathfrak{A}$. For fixed $\rho$ the spectrum of $A(\rho)$ consists only of eigenvalues of finite algebraic multiplicity. We prove that the spectrum of $\mathfrak{A}$ is purely essential and has a certain skeleton structure, i.e., it lies on the imaginary axis and on infinitely many horizontal segments crossing the imaginary axis. In Section 4 numerical calculations show, in accordance with results in [3], that the spectrum of $\mathfrak{A}$ is connected (see Figure 2). Finally, in Section 5, we consider a slight modification of the physical problem, changing the sign of one of the entries in $N$. In this case we show that the spectrum of the corresponding operator has a similar structure, but it has infinitely many components.

\section{The Relation Between the spectra of $\mathfrak{A}$ And $A(\rho)$}

We consider the operators $\mathfrak{A}$ and $A(\rho)$ defined by (1.1) and (1.2). Using standard techniques it is easy to see that the operators $\mathfrak{A}$ and $A(\rho)$ are closed. Indeed, it is well known that the first operator in (1.1) and in (1.2), respectively, is closed, and $N$ and $N(\rho, \cdot)$, respectively, are bounded multiplication operators on the respective Lebesgue spaces. Observe that since $N(\rho, \psi)$ has a continuous extension to $\rho=0$ and $\rho=\infty, A(\rho)$ is also defined and closed for $\rho=0$ and $\rho=\infty$.

In the following the spectrum of an operator $T$ is denoted by $\sigma(T)$ and $\sigma_{p}(T)$ denotes its point spectrum, i. e., the set of its eigenvalues.

Theorem 2.1. The spectra of the operator $\mathfrak{A}$ and the operator family $A$ are related as follows:

$$
\sigma(\mathfrak{A})=\bigcup_{\rho \in[0, \infty]} \sigma(A(\rho))
$$


Proof. For convenience we set $\mathfrak{H}:=L^{2}\left((0, \infty), \rho, L^{2}(0,2 \pi)^{2}\right)$ and $H:=L^{2}(0,2 \pi)^{2}$. Let $\lambda \in \bigcup_{\rho \in[0, \infty]} \sigma(A(\rho))$. Hence there is a $\rho_{0} \in[0, \infty]$ such that $\lambda \in \sigma\left(A\left(\rho_{0}\right)\right)$. Then the range of $A\left(\rho_{0}\right)-\lambda$ is closed and a proper subspace of $H$, or there is a sequence $\left(f_{n}\right)_{1}^{\infty} \subset D,\left\|f_{n}\right\|_{H}=1$, with $\left\|\left(A\left(\rho_{0}\right)-\lambda\right) f_{n}\right\|_{H}<\frac{1}{n}$. We first consider the second case. Since $N$ is continuous, we can choose a sequence of open neighbourhoods $U_{n}$ of $\rho_{0}$ in $[0, \infty)$ so that $\left\|A(\rho)-A\left(\rho_{0}\right)\right\|<\frac{1}{n}$ for all $\rho \in U_{n}$. Let $E_{n} \subset U_{n} \cap(0, \infty)$ be a compact interval with $\beta_{n}:=\int_{E_{n}} \rho d \rho>0, \alpha_{n}:=\left(\beta_{n}\right)^{-\frac{1}{2}}$, and

$$
\mathfrak{f}_{n}(\rho):=\alpha_{n} \chi_{E_{n}}(\rho) f_{n}, \quad \rho \in(0, \infty) .
$$

Obviously, $\mathfrak{f}_{n} \in \mathfrak{H}$ and $\left\|\mathfrak{f}_{n}\right\|_{\mathfrak{H}}=1$. Furthermore, by Fubini's theorem,

$$
\begin{aligned}
\left\|(\mathfrak{A}-\lambda) \mathfrak{f}_{n}\right\|_{\mathfrak{H}}^{2} & =\alpha_{n}^{2} \int_{E_{n}}\left\|(A(\rho)-\lambda) f_{n}\right\|_{H}^{2} \rho d \rho \\
& \leq \alpha_{n}^{2} \beta_{n} \sup _{\rho \in E_{n}}\left(\left\|A(\rho)-A\left(\rho_{0}\right)\right\|+\left\|\left(A\left(\rho_{0}\right)-\lambda\right) f_{n}\right\|_{H}\right)^{2}<\frac{4}{n^{2}},
\end{aligned}
$$

which proves $\lambda \in \sigma(\mathfrak{A})$.

Now assume that $A\left(\rho_{0}\right)-\lambda$ has a closed range which is a proper subspace of $H$. Then there is a $g \in H,\|g\|_{H}=1$, such that $\left(\left(A\left(\rho_{0}\right)-\lambda\right) f, g\right)_{H}=0$ for all $f \in D$. Suppose that $\lambda \notin \sigma(\mathfrak{A})$. Choose $E_{n}$ and $\alpha_{n}$ as above and set

$$
\mathfrak{g}_{n}(\rho):=\alpha_{n} \chi_{E_{n}}(\rho) g, \quad \rho \in(0, \infty) .
$$

Then $\mathfrak{g}_{n} \in \mathfrak{H},\left\|\mathfrak{g}_{n}\right\|_{\mathfrak{H}}=1$, but for $\mathfrak{f}_{n}:=(\mathfrak{A}-\lambda)^{-1} \mathfrak{g}_{n}$ we have

$$
\begin{aligned}
1 & =\left((\mathfrak{A}-\lambda) \mathfrak{f}_{n}, \mathfrak{g}_{n}\right)_{\mathfrak{H}} \\
& =\int_{0}^{\infty}\left((\mathfrak{A}-\lambda) \mathfrak{f}_{n}(\rho), \mathfrak{g}_{n}(\rho)\right)_{H} \rho d \rho \\
& =\alpha_{n} \int_{E_{n}}\left((A(\rho)-\lambda) \mathfrak{f}_{n}(\rho), g\right)_{H} \rho d \rho \\
& =\alpha_{n} \int_{E_{n}}\left(\left(A(\rho)-A\left(\rho_{0}\right)\right) \mathfrak{f}_{n}(\rho), g\right)_{H} \rho d \rho \\
& \leq \alpha_{n} \int_{E_{n}}\left\|A(\rho)-A\left(\rho_{0}\right)\right\|\left\|\mathfrak{f}_{n}(\rho)\right\|_{H} \rho d \rho \\
& <\alpha_{n} \frac{1}{n} \int_{E_{n}}\left\|\mathfrak{f}_{n}(\rho)\right\|_{H} \rho d \rho \\
& \leq \alpha_{n} \frac{1}{n} \beta_{n}^{\frac{1}{2}}\left\|\mathfrak{f}_{n}\right\|_{\mathfrak{H}} \\
& =\frac{1}{n}\left\|\mathfrak{f}_{n}\right\|_{\mathfrak{H}},
\end{aligned}
$$

and hence $\left\|\mathfrak{f}_{n}\right\|_{\mathfrak{H}}=\left\|(\mathfrak{A}-\lambda)^{-1} \mathfrak{g}_{n}\right\|_{\mathfrak{H}} \rightarrow \infty$ as $n \rightarrow \infty$. This is a contradiction because $(\mathfrak{A}-\lambda)^{-1}$ is bounded by assumption. 
Conversely, let $\lambda \in \sigma(\mathfrak{A})$. If $\lambda$ is an eigenvalue of $\mathfrak{A}$, then there exists a non-zero $\mathfrak{f} \in D(\mathfrak{A})$ such that $\mathfrak{A} \mathfrak{f}=\lambda \mathfrak{f}$. Hence, by $(1.3), A(\rho) \mathfrak{f}(\rho)=\lambda \mathfrak{f}(\rho)$ for almost all $\rho \in(0, \infty)$. Since $\mathfrak{f} \neq 0, \mathfrak{f}(\rho) \neq 0$ for all $\rho$ in some set of positive measure. Thus there is a $\rho_{0} \in(0, \infty)$ such that $\mathfrak{f}\left(\rho_{0}\right) \neq 0$ and $A\left(\rho_{0}\right) \mathfrak{f}\left(\rho_{0}\right)=\lambda \mathfrak{f}\left(\rho_{0}\right)$. This proves

$$
\sigma_{p}(\mathfrak{A}) \subset \bigcup_{\rho \in(0, \infty)} \sigma(A(\rho)) .
$$

Now assume that $\lambda \in \sigma(\mathfrak{A})$ is not an eigenvalue of $\mathfrak{A}$. Then $\mathfrak{A}-\lambda$ is injective but not surjective, and we can find an element $\mathfrak{g} \in \mathfrak{H}$ such that $(\mathfrak{A}-\lambda) \mathfrak{f} \neq \mathfrak{g}$ for all $\mathfrak{f} \in D(\mathfrak{A})$. Assume $\lambda \notin \bigcup_{\rho \in[0, \infty]} \sigma(A(\rho))$. For (almost all) $\rho \in(0, \infty)$ we define

$$
\mathfrak{h}(\rho):=(A(\rho)-\lambda)^{-1} \mathfrak{g}(\rho) .
$$

From the continuity of the inversion, see [2, Theorem IV.1.16], it follows that the mapping $\rho \mapsto(A(\rho)-\lambda)^{-1}$ from $[0, \infty]$ into the bounded operators on $H$ is continuous. Hence $\mathfrak{h}$ is measurable and

$$
\left(\int_{0}^{\infty}\|\mathfrak{h}(\rho)\|_{H}^{2} \rho d \rho\right)^{\frac{1}{2}} \leq \sup _{\rho \in[0, \infty]}\left\|(A(\rho)-\lambda)^{-1}\right\|\|\mathfrak{g}\|_{\mathfrak{H}}<\infty .
$$

Thus $\mathfrak{h} \in \mathfrak{H}$, and $\mathfrak{h}(\rho) \in D$ clearly implies $\mathfrak{h} \in D(\mathfrak{A})$. This yields the contradiction $(\mathfrak{A}-\lambda) \mathfrak{h}=\mathfrak{g}$.

\section{The Calculation of $\sigma(\mathfrak{A})$}

Theorem 3.1. The operator $\mathfrak{A}$ has no eigenvalues and there are constants $a \geq 0$ and $b \geq 0$ such that

$\sigma(\mathfrak{A})=\mathrm{i} \mathbb{R} \cup\{\lambda \in \mathbb{C}:|\Re(\lambda)| \leq a, \Im(\lambda) \in \mathbb{Z}\} \cup\left\{\lambda \in \mathbb{C}:|\Re(\lambda)| \leq b, \Im(\lambda) \in \frac{1}{2}+\mathbb{Z}\right\}$

or constants $c \geq 0$ and $d<\frac{1}{2}$ such that

$$
\sigma(\mathfrak{A})=\bigcup_{k \in \mathbb{Z}} \mathrm{i}[k-c, k+c] \cup\{\lambda \in \mathbb{C}:|\Re(\lambda)| \leq d, \Im(\lambda) \in \mathbb{Z}\} .
$$

Proof. To find the spectrum of $\mathfrak{A}$, by Theorem 2.1 it is sufficient to know $\sigma(A(\rho))$ for $\rho \in[0, \infty]$. We will show that the eigenvalue problem for $A(\rho)$ is equivalent to an eigenvalue problem for a second order differential equation.

It is well known from Floquet theory, see [1, Chapter 1] or [6, Chapter II], that the spectrum of $A(\rho)$ is discrete. Let $\lambda \in \sigma(A(\rho))$ and let $y$ be an eigenfunction of $A(\rho)$ at $\lambda$. Put $u(\psi):=\mathrm{e}^{-\lambda \psi} y(\psi)$. Then $u(2 \pi)=\mathrm{e}^{-2 \lambda \pi} y(2 \pi)=\mathrm{e}^{-2 \lambda \pi} y(0)=$ $\mathrm{e}^{-2 \lambda \pi} u(0)$ and

$$
\begin{aligned}
u^{\prime}(\psi)+2 N(\rho, \psi) u(\psi) & =-\lambda \mathrm{e}^{-\lambda \psi} y(\psi)+\mathrm{e}^{-\lambda \psi} y^{\prime}(\psi)+2 N(\rho, \psi) \mathrm{e}^{-\lambda \psi} y(\psi) \\
& =-\lambda \mathrm{e}^{-\lambda \psi} y(\psi)+\lambda \mathrm{e}^{-\lambda \psi} y(\psi) \\
& =0
\end{aligned}
$$


Since these steps can be reversed, $\lambda \in \sigma(A(\rho))$ if and only if the problem

$$
y^{\prime}+2 N(\rho, \cdot) y=0, \quad y(2 \pi)=\mathrm{e}^{-2 \lambda \pi} y(0)
$$

has a nontrivial solution. Now let $\rho \in[0, \infty)$. With

$$
w(\psi):=\frac{\rho^{2}(1-\delta \cos (2 \psi))+1-\delta^{2}}{2\left(1-\delta^{2}\right)}
$$

we have

$$
2 N=\left(\begin{array}{rr}
0 & -\frac{1}{w} \\
2 & \frac{w^{\prime}}{w}
\end{array}\right) .
$$

Then, with $y=\left(y_{1}, y_{2}\right)^{\top}$, the equation $y^{\prime}+2 N y=0$ can be written in the form

$$
\begin{aligned}
y_{1}^{\prime}-\frac{1}{w} y_{2} & =0, \\
y_{2}^{\prime}+2 y_{1}+\frac{w^{\prime}}{w} y_{2} & =0 .
\end{aligned}
$$

This is equivalent to

$$
\begin{aligned}
w^{2} y_{1}^{\prime}-w y_{2} & =0, \\
\left(w y_{2}\right)^{\prime}+2 w y_{1} & =0 .
\end{aligned}
$$

Differentiating the first equation and inserting into the second one gives

$$
\left(w^{2} y_{1}^{\prime}\right)^{\prime}+2 w y_{1}=0 \text {. }
$$

Since $w$ is $2 \pi$-periodic, the boundary conditions for $y_{1}$ are

$$
y_{1}(\pi)=\mathrm{e}^{-2 \lambda \pi} y_{1}(0), \quad y_{1}^{\prime}(\pi)=\mathrm{e}^{-2 \lambda \pi} y_{1}^{\prime}(0) .
$$

If now $y_{1}$ is a (nonzero) solution of this problem, then $y_{2}=w y_{1}^{\prime}$ gives a nonzero solution $y=\left(y_{1}, y_{2}\right)^{\top}$ of (3.1). If, conversely, (3.1) has a nonzero solution, then $y_{1} \neq 0$ since $y_{1}=0$ would imply $y_{2}=0$. This shows that $\lambda \in \sigma(A(\rho))$ if and only if (3.2), (3.3) has a nontrivial solution.

Let $y_{11}, y_{12}$ be the fundamental system of $(3.2)$ with $y_{11}(0)=1, y_{11}^{\prime}(0)=0$, $y_{12}(0)=0, y_{12}^{\prime}(0)=1$. It is a well-known fact that the Wronski determinant $w^{2}\left(y_{11} y_{12}^{\prime}-y_{11}^{\prime} y_{12}\right)$ is constant, and in particular

$$
\operatorname{det}\left(\begin{array}{ll}
y_{11}(2 \pi) & y_{12}(2 \pi) \\
y_{11}^{\prime}(2 \pi) & y_{12}^{\prime}(2 \pi)
\end{array}\right)=1
$$

The existence of a nonzero solution of the boundary value problem (3.2), (3.3) means that

$$
\operatorname{det}\left(\begin{array}{cc}
y_{11}(2 \pi)-\mathrm{e}^{-2 \lambda \pi} & y_{12}(2 \pi) \\
y_{11}^{\prime}(2 \pi) & y_{12}^{\prime}(2 \pi)-\mathrm{e}^{-2 \lambda \pi}
\end{array}\right)=0 .
$$

This leads to

$$
\mathrm{e}^{-4 \lambda \pi}-\mathrm{e}^{-2 \lambda \pi}\left(y_{11}(2 \pi)+y_{12}^{\prime}(2 \pi)\right)+1=0 .
$$


Observe that we can find the spectrum of $A(0)$ explicitly since $w(0, \psi)=\frac{1}{2}$. Here $y_{11}(\psi)=\cos (2 \psi)$ and $y_{12}(\psi)=\frac{1}{2} \sin (2 \psi)$, and the solutions of (3.5) are $\lambda=\mathrm{i} k, k \in \mathbb{Z}$.

The two solutions of (3.5), in terms of $\mathrm{e}^{-2 \lambda \pi}$, are two positive numbers whose product is 1 , two negative numbers whose product is 1 , or two conjugate complex numbers with modulus 1 . This means that $\lambda$ lies in $\mathbb{R}+\mathrm{i} \mathbb{Z}$, in $\mathbb{R}+\mathrm{i}(1 / 2+\mathbb{Z})$, or in $i \mathbb{R}$. The continuous dependence of the spectrum on $\rho$ and the fact that the spectrum for $\rho=0$ lies on the imaginary axis shows that the parts of the spectrum on the parallels to the real axis must be intervals. The two different representations given in the statement of this theorem depend on whether or not there is $\rho \in[0, \infty]$ such that $y_{11}(2 \pi)+y_{12}^{\prime}(2 \pi) \leq-2$.

To show that $\sigma_{p}(\mathfrak{A})=\emptyset$, observe that the characteristic determinant $\Delta$ is not unique, but one can choose $\Delta$ such that it depends analytically on the spectral parameter $\lambda$. Since $N$ depends analytically on $\rho, \Delta$ can be chosen so that it depends analytically on both parameters. If $\lambda$ were an eigenvalue of $\mathfrak{A}$, then $\lambda \in \sigma(A(\rho))$ for $\rho$ in a set of positive Lebesgue measure, see the proof of Theorem 2.1. In that case, $\Delta(\rho, \lambda)=0$ for this particular $\lambda$ and a set of $\rho$ with limit point in $(0, \infty)$. By the identity theorem for analytic functions, this would imply that $\Delta(\rho, \lambda)=0$ for all $\rho \in(0, \infty)$. This contradicts the fact that the eigenvalues are not constant as functions of $\rho$, which will follow from the considerations in the next section.

\section{Numerical RESUlts FOR $\sigma(\mathfrak{A})$}

Below we will show by numerical calculations, in accordance with [3], that for the spectrum of $\mathfrak{A}$ described in Theorem 3.1 the first case with $b=0$ applies, i. e.,

$$
\sigma(\mathfrak{A})=\mathrm{i} \mathbb{R} \cup\{\lambda \in \mathbb{C}:|\Re(\lambda)| \leq a, \Im(\lambda) \in \mathbb{Z}\}
$$

We want to investigate how the solutions of (3.2) depend on $\rho$ and thus find information about the dependence of the eigenvalues of $A(\rho)$ on $\rho$. First let us consider $\rho$ near 0 . For this it is easier to introduce $\tau=\rho^{2}$. With

$$
w_{1}(\psi):=\frac{1-\delta \cos (2 \psi)}{2\left(1-\delta^{2}\right)}
$$

we have $w(\psi)=\tau w_{1}(\psi)+\frac{1}{2}$. Let $y_{1}$ be a solution (depending on $\tau$ ) of (3.2) such that $y_{1}(0)$ and $y_{1}^{\prime}(0)$ are independent of $\tau$. Then we can differentiate (3.2) with respect to $\tau$ and obtain (with $\frac{\partial}{\partial \tau} y_{1}=: y_{1 \tau}$ ) that

$$
\left(\left(\frac{\partial}{\partial \tau} w^{2}\right) y_{1}^{\prime}\right)^{\prime}+\left(w^{2} y_{1 \tau}^{\prime}\right)^{\prime}+2\left(\frac{\partial}{\partial \tau} w\right) y_{1}+2 w y_{1 \tau}=0
$$

Evaluation at $\tau=0$ gives

$$
\frac{1}{4} y_{1 \tau}^{\prime \prime}+y_{1 \tau}=-\left(w_{1} y_{1}^{\prime}\right)^{\prime}-2 w_{1} y_{1}
$$


Note that $y_{11}(\psi)=\cos (2 \psi), y_{12}(\psi)=\frac{1}{2} \sin (2 \psi)$ at $\tau=0$ and that $y_{1 \tau}(0)=0$, $y_{1 \tau}^{\prime}(0)=0$ since the initial conditions do not depend on $\tau$. An evaluation of $y_{11 \tau}$ and $y_{12 \tau}$ (e.g., with MAPLE) shows that $y_{11 \tau}(\pi)+y_{12 \tau}^{\prime}(\pi)=0$ at $\tau=0$. Hence we need the second derivative with respect to $\tau$. This gives

$$
\left(w^{2} y_{1 \tau \tau}^{\prime}\right)^{\prime}+4\left(w\left(\frac{\partial}{\partial \tau} w\right) y_{1 \tau}^{\prime}\right)^{\prime}+2\left(w_{1}^{2} y_{1}^{\prime}\right)^{\prime}+4 w_{1} y_{1 \tau}+2 w y_{1 \tau \tau}=0
$$

and thus, at $\tau=0$,

$$
\frac{1}{4} y_{1 \tau \tau}^{\prime \prime}+y_{1 \tau \tau}=-2\left(w_{1} y_{1 \tau}^{\prime}\right)^{\prime}-2\left(w_{1}^{2} y_{1}^{\prime}\right)^{\prime}-4 w_{1} y_{1 \tau}
$$

Another calculation (e.g., with MAPLE) gives

$$
y_{11 \tau \tau}(2 \pi)+y_{12 \tau \tau}^{\prime}(2 \pi)=-\frac{8 \pi^{2}}{\left(1-\delta^{2}\right)^{2}}
$$

at $\tau=0$. Hence $-2<y_{11}(2 \pi)+y_{12}^{\prime}(2 \pi)<2$ for sufficiently small positive $\rho$, and the eigenvalue equation (3.5) shows that the eigenvalues of $A(\rho)$ for these $\rho$ lie on the imaginary axis.

We have

$$
N(\infty, \cdot)=\left(\begin{array}{cc}
0 & 0 \\
2 & \frac{w_{\infty}^{\prime}}{w_{\infty}}
\end{array}\right)
$$

where $w_{\infty}(\psi)=1-\delta \cos (2 \psi)$. Putting

$$
W_{\infty}(\psi):=\int_{0}^{\psi} \frac{1}{w_{\infty}(t)} d t
$$

it is easy to see that

$$
Y(\psi)=\left(\begin{array}{cc}
\mathrm{e}^{\lambda \psi} & 0 \\
2 w_{\infty}(\psi) W_{\infty}(\psi) \mathrm{e}^{\lambda \psi} & w_{\infty}(\psi) \mathrm{e}^{\lambda \psi}
\end{array}\right)
$$

is a fundamental matrix of $y^{\prime}+2 N(\infty, \cdot) y=\lambda y$, and therefore

$$
\Delta(\lambda)=\operatorname{det}\left(\begin{array}{cc}
\mathrm{e}^{2 \lambda \pi}-1 & 0 \\
2 w_{\infty}(2 \pi) W_{\infty}(2 \pi) \mathrm{e}^{2 \lambda \pi} & w_{\infty}(2 \pi)\left(\mathrm{e}^{2 \lambda \pi}-1\right)
\end{array}\right)
$$

is a characteristic determinant of $A(\infty)$. Obviously, $\Delta(\lambda)$ is zero if and only if $\lambda \in \mathrm{i} \mathbb{Z}$. Hence, at $\rho=\infty, y_{11}(2 \pi)+y_{12}^{\prime}(2 \pi)=2$.

In order to investigate the behaviour of the eigenvalues for $\rho$ near $\infty$, we now make the substitution $\tau=\rho^{-2}$, so that, after multiplication by $\tau^{2},(5.1)$ becomes (we already know that the eigenvalues depend continuously on $\rho$ at $\rho=\infty$, so any formulation of the eigenvalue problem which is continuous at $\tau=0$ is acceptable)

$$
\left(\left(w_{1}+\frac{\tau}{2}\right)^{2} y_{1}^{\prime}\right)^{\prime}+\tau\left(2 w_{1}+\tau\right) y_{1}=0
$$


Differentiation with respect to $\tau$ leads to

$$
\left(\left(w_{1}+\frac{\tau}{2}\right)^{2} y_{1 \tau}^{\prime}\right)^{\prime}+\left(\left(w_{1}+\frac{\tau}{2}\right) y_{1}^{\prime}\right)^{\prime}+2\left(w_{1}+\tau\right) y_{1}+\tau\left(2 w_{1}+\tau\right) y_{1 \tau}=0 .
$$

At $\tau=0$ we obtain

$$
\left(w_{1}^{2} y_{1 \tau}^{\prime}\right)^{\prime}=-\left(w_{1} y_{1}^{\prime}\right)^{\prime}-2 w_{1} y_{1}
$$

where

$$
\left(w_{1}^{2} y_{1}^{\prime}\right)^{\prime}=0
$$

Hence $w_{1}^{2} y_{1}^{\prime}$ is constant. From $y_{11}^{\prime}(0)=0$ it therefore follows that $y_{11}^{\prime}=0$, and hence $y_{11}(\psi)=1$. For $y_{12}$ we get $w_{1}^{2} y_{12}^{\prime}=w_{1}^{2}(0)$, and hence

$$
y_{12}(\psi)=\int_{0}^{\psi} \frac{w_{1}^{2}(0)}{w_{1}^{2}(t)} d t
$$

Thus

$$
\left(w_{1}^{2} y_{11 \tau}^{\prime}\right)^{\prime}=-2 w_{1}
$$

and

$$
w_{1}^{2}(\psi) y_{12 \tau}^{\prime}(\psi)=-\frac{w_{1}^{2}(0)}{w_{1}(\psi)}+w_{1}(0)-2 \int_{0}^{\psi} w_{1}(t) y_{12}(t) d t .
$$

This gives

$$
y_{12 \tau}^{\prime}(2 \pi)=-2 \int_{0}^{2 \pi} w_{1}(\psi) \int_{0}^{\psi} \frac{1}{w_{1}^{2}(t)} d t d \psi=-2 \int_{0}^{2 \pi} \frac{1}{w_{1}^{2}(t)} \int_{t}^{2 \pi} w_{1}(\psi) d \psi d t
$$

and

which leads to

$$
w_{1}^{2}(t) y_{11 \tau}^{\prime}(t)=-2 \int_{0}^{t} w_{1}(\psi) d \psi
$$

$$
y_{11 \tau}(2 \pi)=-2 \int_{0}^{2 \pi} \frac{1}{w_{1}^{2}(t)} \int_{0}^{t} w_{1}(\psi) d \psi d t
$$

Therefore

$$
y_{11 \tau}(2 \pi)+y_{12 \tau}^{\prime}(2 \pi)=-2 \int_{0}^{2 \pi} \frac{1}{w_{1}^{2}(t)} \int_{0}^{2 \pi} w_{1}(\psi) d \psi d t<0,
$$

and hence $-2<y_{11}(2 \pi)+y_{12}^{\prime}(2 \pi)<2$ for sufficiently large $\rho$. This shows that the eigenvalues of $A(\rho)$ for these $\rho$ lie on the imaginary axis.

To summarize, we have shown that the eigenvalues of $A(\rho)$ lie on the imaginary axis for $\rho$ near 0 and $\infty$.

For $\delta=0$ we can solve (3.2) explicitly. Here

$$
y_{11}(2 \pi)+y_{12}^{\prime}(2 \pi)=2 \cos \left(\frac{4 \pi}{\sqrt{\rho^{2}+1}}\right) \text {, }
$$


and the eigenvalue equation (3.5) becomes

$$
\left(\exp (-2 \lambda \pi)-\exp \left(\frac{4 \pi \mathrm{i}}{\sqrt{\rho^{2}+1}}\right)\right)\left(\exp (-2 \lambda \pi)-\exp \left(-\frac{4 \pi \mathrm{i}}{\sqrt{\rho^{2}+1}}\right)\right)=0
$$

and therefore $\sigma(A(\rho))=\mathrm{i}\left( \pm \frac{2}{\sqrt{\rho^{2}+1}}+\mathbb{Z}\right)$, whence $\sigma(\mathfrak{A})=\mathrm{i} \mathbb{Z}$.

For $\delta>0$ it seems to be impossible to obtain more exact information on the spectrum of $A(\rho)$. Therefore we present some numerical results.

Figure 1 shows the values of $y_{11}(2 \pi)+y_{12}^{\prime}(2 \pi)$. The different curves correspond to the values of $\delta=0,0.1, \ldots, 0.6$ and are plotted as functions of $\frac{\rho^{2}}{\rho^{2}+1}$. The curves are "decreasing" near $\rho=0$ and $\rho=\infty$ with increasing $\delta$, but their maxima increase with $\delta$.

Note that the eigenvalues of $A(\rho)$ lie on the imaginary axis if $y_{11}(2 \pi)+y_{12}^{\prime}(2 \pi)$ lies between -2 and 2 , whereas they move onto the lines $\mathrm{i} k+\mathbb{R}, k \in \mathbb{Z}$, if $y_{11}(2 \pi)+y_{12}^{\prime}(2 \pi)$ becomes greater than 2 .

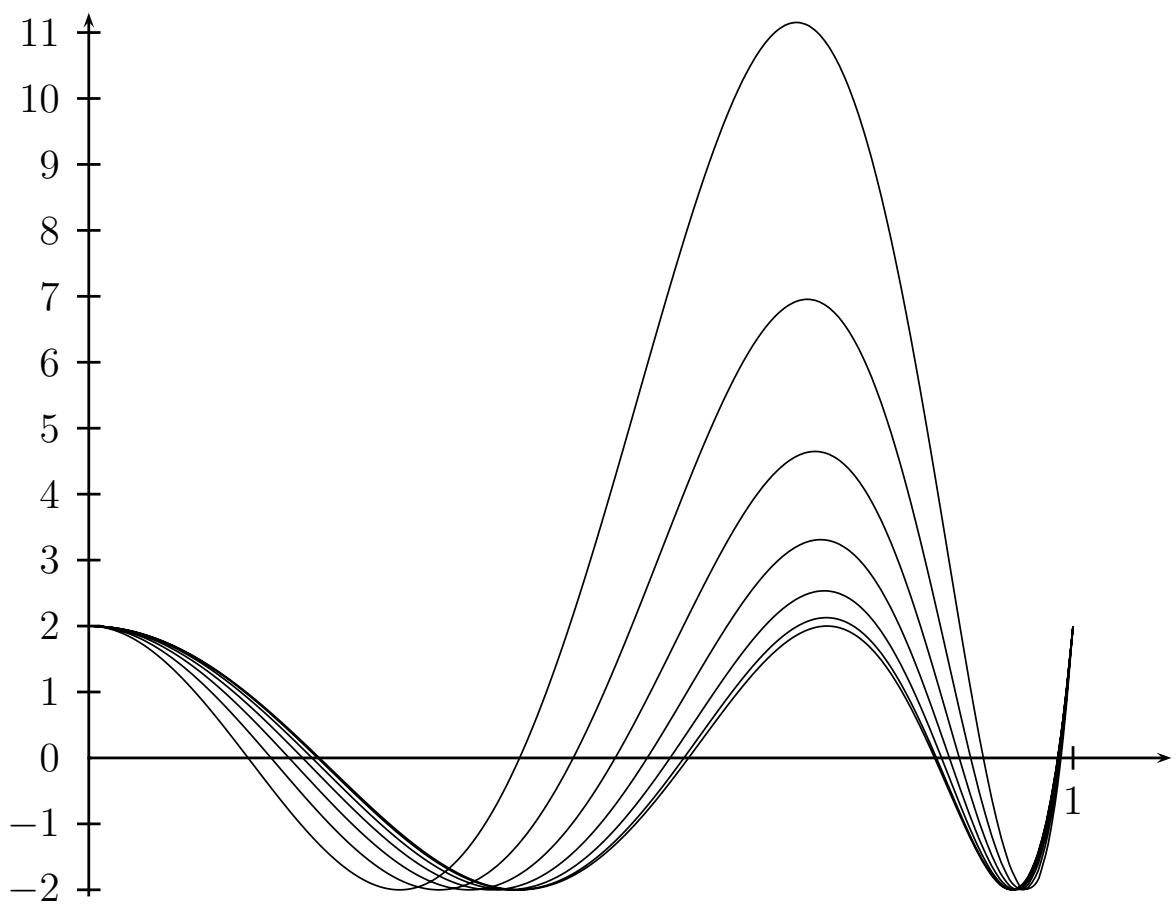

Figure 1: The values of $y_{11}(2 \pi)+y_{12}^{\prime}(2 \pi)$ as function of $\frac{\rho^{2}}{\rho^{2}+1}$

The differential equations were solved with the Bulirsch-Stoer method, using the implementation [4, Section 15.4]. The above curves suggest that the spectrum of $\mathfrak{A}$ is the union of the imaginary axis and lines $\mathrm{i} k+[-a, a]$, where $a$ is strictly 
increasing with $\delta$ and $a=0$ at $\delta=0$. Numerical calculations of the part of the spectrum of $\mathfrak{A}$ with imaginary parts between -2 and 2 for $\delta=0.6$ and $\delta=0.9$ are shown in Figure 2.
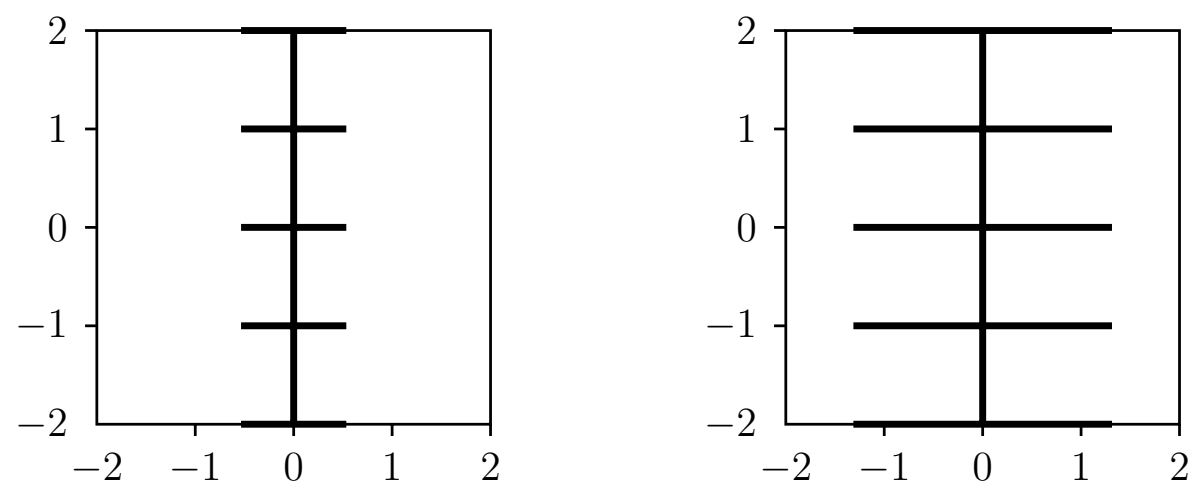

Figure 2: $\sigma(\mathfrak{A})$ for $\delta=0.6$ and $\delta=0.9$

\section{A MODIFIED PROBLEM}

As in Sections 2-4 we consider an operator of the form

where now

$$
\mathfrak{A}_{+}=\left(\begin{array}{cc}
\frac{\partial}{\partial \psi} & 0 \\
0 & \frac{\partial}{\partial \psi}
\end{array}\right)+2 N_{+}
$$

$$
N_{+}(\rho, \psi)=\left(\begin{array}{cc}
0 & \frac{1-\delta^{2}}{\rho^{2}(1-\delta \cos (2 \psi))+1-\delta^{2}} \\
1 & \frac{\delta \rho^{2} \sin (2 \psi)}{\rho^{2}(1-\delta \cos (2 \psi))+1-\delta^{2}}
\end{array}\right),
$$

that is, in the right upper entry of $N_{+}$the negative of the entry in $N$ considered so far is taken. It is not difficult to see that also the operator $\mathfrak{A}_{+}$is closed.

Theorem 5.1. The operator $\mathfrak{A}_{+}$has no eigenvalues and there is a constant $a \geq 2$ such that

$$
\sigma\left(\mathfrak{A}_{+}\right)=\{\lambda \in \mathbb{C}:|\Re(\lambda)| \leq a, \Im(\lambda) \in 2 \mathbb{Z}\} .
$$

Proof. The proof is very similar to that of Theorem 3.1, and we only point out the differences. Here $w$ has to be replaced by $-w$, and therefore (3.2) becomes

$$
-\left(w^{2} y_{1}^{\prime}\right)^{\prime}+2 w y_{1}=0
$$

and again (3.5) becomes the eigenvalue equation.

From (5.1) it follows that $w^{2} y_{1 j}^{\prime}$ is strictly increasing at a point if $y_{1 j}$ is positive there $(j=1,2)$. Then the initial conditions imply that both $y_{11}$ and $y_{12}$ together with their derivatives are positive on $(0,2 \pi]$. In particular, (3.4) implies that 
$y_{11}(2 \pi) y_{12}^{\prime}(2 \pi)>1$, and the arithmetic-geometric mean inequality yields that the positive numbers $y_{11}(2 \pi)$ and $y_{12}^{\prime}(2 \pi)$ satisfy $y_{11}(2 \pi)+y_{12}^{\prime}(2 \pi)>2$. This shows that the two solutions of (3.5) as a quadratic equation in $\mathrm{e}^{-2 \lambda \pi}$ are positive and inverses of each other. Hence there is a positive function $\alpha:[0, \infty) \rightarrow(0, \infty)$ such that the eigenvalues of $A(\rho)$ are $\{ \pm \alpha(\rho)+\mathrm{i} k: k \in \mathbb{R}\}$. Performing the differentiation in (5.1) and dividing by $w^{2}$ one obtains a differential equation whose coefficient at $y^{\prime \prime}$ is 1 and whose other coefficients are continuous functions of $\rho \in[0, \infty)$. Hence also $y_{11}(2 \pi)$ and $y_{12}^{\prime}(2 \pi)$ depend continuously on $\rho \in[0, \infty)$. Therefore, $\alpha$ is continuous and has a continuous extension to $\rho \in[0, \infty]$.

Since $A(\infty)$ is as in Section 4 , it follows that $\alpha(\infty)=0$. Thus $\alpha([0, \infty])$ is a (compact) interval containing the points 0 and 2 . Hence $\alpha([0, \infty]) \cup(-\alpha([0, \infty]))$ is a compact interval of the form $[-a, a]$, and the statement on $\sigma(\mathfrak{A})$ follows.

That $\mathfrak{A}_{+}$has no eigenvalues follows as in Section 3 from the fact that $\alpha$ is not constant.

\section{REFERENCES}

[1] Eastham, M. S. P., The spectral theory of periodic differential equations, Scottish Academic Press, Edinburgh, 1973.

[2] Kato, T., Perturbation theory for linear operators, $2^{\text {nd }}$ ed., Springer-Verlag, Berlin, 1980.

[3] Lifschitz, A., Exact description of the spectrum of elliptical vortices in hydrodynamics and magnetohydrodynamics, Phys. Fluids 7:7 (1995), 1626-1636.

[4] Press, W. H., et al., Numerical Recipes in PASCAL, Cambridge University Press, 1989.

[5] Reed, M., Simon, B., Analysis of operators, IV, Academic Press, New York, 1978.

[6] Yakubovich, V. A., Starzhinskii, V. M., Linear differential equations with periodic coefficients, Part 1, John Wiley \& Sons, New York, 1975.

NWF I - Mathematik, University of Regensburg, D-93040 Regensburg, Germany, robert.denk@mathematik. uni-regensburg. de

Dept. of Mathematics, University of the Witwatersrand, 2050 WITS, South Africa, 036man@cosmos.wits.ac.za

Dept. of Mathematics and Computer Science, University of Leicester, University Road, Leicester LE1 7RH, United Kingdom, c.tretter@mcs.le.ac.uk 